\title{
Quarks, Bootstraps and Monads
}

\author{
David M. Harrison \\ Department of Physics \\ University of Toronto
}

In Physics, there are two main threads in our search for the ultimate constituents of the world. They are in some senses in competition. One thread views the world as being made of atoms, while the other views the world as being made of relationships. One or the other of these worldviews is found in other contexts, including the ancient Greeks, Hindu and Buddhist philosophy, and Chinese classifications of the world. I review these two approaches and their historical and cross-cultural reflections. The view of the world being made of relationships is found to be echoed in Leibnizian thought. This echo may not be a coincidence or even an archetype, but may have an historical cause.

\section{Introduction}

Most introductory Physics textbooks present the atomistic worldview as a fact, and usually discuss the related "fact" that the world is made of elementary particles: electrons, quarks and neutrinos. A central mystery of this story, often ignored, is that the quarks are unobservable. Various theories have been proposed to explain why we can't ever isolate a quark. It turns out that atomistic conceptions with the most elementary least part being unobservable also appears in the thinking of Democritus, Plato, and the Sankhya Hindus.

However, there is a parallel storyline concentrating on the idea that the world is made of relationships. Standard textbooks often ignore or downplay this alternative worldview. In Physics this approach is often called the bootstrap, or more recently string theory. It shares key features with concepts from Anaxagoras, Leibniz, the Buddhists, and the Chinese.

The official storyline, then, traces the atomistic worldview in the Western scientific tradition from Newton to Dalton to Gell-Mann. The parallel one traces its roots from Leibniz to Mach to Chew. The fact that the former trio of names is much better known than the latter is an artifact of the culture of our scientific community.

In this paper, I will sketch in both storylines and make some connections to other cultures. Two secondary themes will be woven into the discussion: the role of mind in classifications of the world, and the number zero. I save a discussion of Leibniz' role in all this to the final section. 


\section{Elementary Particle Physics and High Energy Physics}

Traditionally a single research field of Physics has been named either High Energy Physics or Elementary Particle Physics. These two terms are often treated as synonyms. The first designation arises because experimentally high energies are necessary to investigate the question of what the universe is made at its most fundamental level. The second phrase assumes the answer: the world is made of elementary particles.

In this section I will first give a very brief overview of Elementary Particle Physics, and will show that at least one of its most basic concepts is also reflected in ancient Greek atomism, Platonic classifications, and in Sankhya Hindu ideas about the world. Next I will discuss an approach to these questions that does not think of the world as being made of particles, but instead each of the so-called elementary particles is a reflection of all the others. I will show that this idea, often called the bootstrap in Physics, also appears in both Buddhist and Chinese analyses of the world.

Jungians are adept at finding basic ideas and symbols that transcend a particular culture to discover archetypes, innate universal prototypes of ideas and intuitions shared by all people. Jung and Pauli suggested that our most basic mathematical intuitions may be an example of an archetype. (Jung \& Pauli, von Franz) The fact that the basic ideas of two differing approaches to the ultimate constituents of the world are shared by different cultures, then, may mean that both have an archetypal component.

\section{Classifications of a World Made of Elementary Particles}

\section{Elementary Particle Physics}

The modern concept of the atom goes back to Dalton in 1808, although ideas of the world being particulate also dominated Newton's thinking much earlier. Dalton's idea swept the Western world, and influenced thinking in biology, sociology, psychology, and more. There was opposition to this idea: Mach in particular never accepted the notion of atoms. William Blake's position, written before Dalton, is also clear:

The atoms of Democritus

And Newton's Particles of light

Are sands upon the Red Sea shore,

Where Israel's tents do shine so bright.

It is interesting to note that Dalton believed he had proved the existence of atoms from four assumptions, although each and every one of them is now believed to be factually wrong or was used by Dalton in a logically inconsistent manner (Holton, 1973, p. 385386).

In 1897 J.J. Thomson showed that Dalton's atoms were not elementary: they contained light negatively charged electrons. About ten years later Thomson's student Ernest Rutherford discovered that atoms also contain a massive positively charged nucleus. 
Soon it was determined that the nucleus is made of a number of positively charged protons and electrically neutral neutrons.

In 1935 Yukawa proposed another elementary particle, the meson. It was experimentally observed in 1947. Along the way some "minor" elementary particles were added to the zoo: the particulate aspect of light called the photon, a high-mass electron called a muon, and the nearly massless neutrino; I will ignore these in what follows.

So for a few years the elementary particles of the world were the electron, proton, neutron, mesons, and their corresponding antiparticles. In 1963 Gell-Mann and independently Zweig proposed that the protons, neutrons and mesons were made up of quarks. In this view, then, the protons, neutrons and mesons are not elementary, although the quarks are. Believers in this taxonomy think there are 6 quarks plus their antiparticles,

The next logical question might be of what are the quarks made? So far, the answer seems to be nothing: quarks and electrons are truly elementary point-particles in this view. If a substructure to quarks is ever discovered I propose calling it a smitheron, whose plural is of course smithereens!

Immediately after the quark model was proposed, experimentalists began looking for them. They looked in cosmic rays, nuclear decays, cyclotrons, oysters (true!), basically everywhere they could think of. However, all the attempts ended in failure. So finally, believers came to the conclusion that we can't have an independent single quark, that they are "confined" inside the protons, neutrons, and mesons.

A rough analogy involves magnets. As you may know, magnets have a "North" and "South" pole. If we cut a magnet in half, as indicated to the right, we end up with two little magnets, each with a North and South pole. We have never observed a North or South magnetic pole separate from its opposite pole. Thus if these magnetic monopoles exist, they too are confined.

The idea of objects being made of other more fundamental objects is sometimes called a parton theory, with the parts making up the whole. Since the quarks are confined, quark

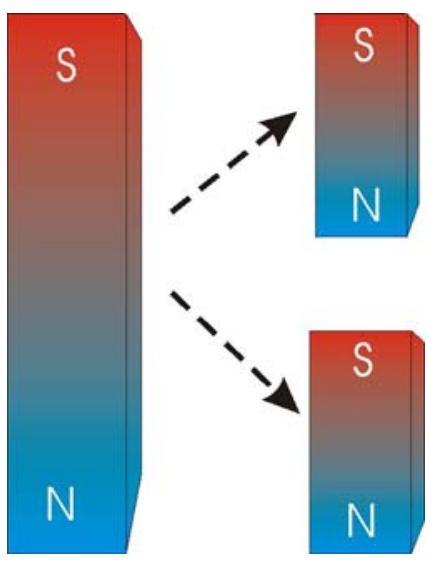
theory is a confined parton model.

\section{Ancient Greek Atomism}

Democritus and Epicurus (4th - 5th century BCE) also believed that the world was made of atoms. The theory is fully described by Lucretius in De Rerum Natura.

The word atom means literally that which can not be divided. Lucretius also used the word elementa to describe them; the word also means the letters of the alphabet. This is interesting since Needham in his classic multi-volume study Science and Civilisation in 
China has speculated that only cultures with alphabetic written languages develop atomistic theories (Volume 4, pg. 13). We shall see in the next sub-section, for example, that the Chinese, who do not have an alphabetic written language, analyzed the world into five elements but those elements are definitely not atoms.

Democritus describes an atomistic worldview in a famous statement:

By convention sweet is sweet, by convention bitter is bitter, by convention hot is hot, by convention cold is cold, by convention color is color. But in reality there are atoms and the void. That is, objects of sense are supposed to be real and it is customary to regard them as such, but in truth they are not. Only the atoms and the void are real.

Lucretius' writing is pretty dense, but notice his discussion of least parts:

The first principles, then, are solid and simple; they consist of least parts tightly compacted and compressed - not compounded by the coming together of these parts, but rather enduring in everlasting simplicity; from them nothing is permitted to be torn away or diminished, by nature which preserves the seed of things. (Humphries trans.)

In 1977 Julia H. Gaisser and T.K. Gaisser realised that the least parts that Lucretius writes about are a confined parton just as are the quarks. They also suggested that the reason for the need for confined partons in atomistic theories is that there is "an inherent conflict between the intuitive notion of infinite divisibility and the idea of discrete fundamental atoms of matter."

\section{Platonic Classifications}

Plato, of course, was not an atomist. He did, however, accept Empedocles' notion that there are four fundamental elements: fire, earth, air and water. He also knew that there are five and only five regular convex polyhedra each of whose faces are all identical: the tetrahedron, cube, octahedron, icosahedron and dodecahedron. In his one work on science, Timaeus, Plato associated four of these polyhedra with the four elements.
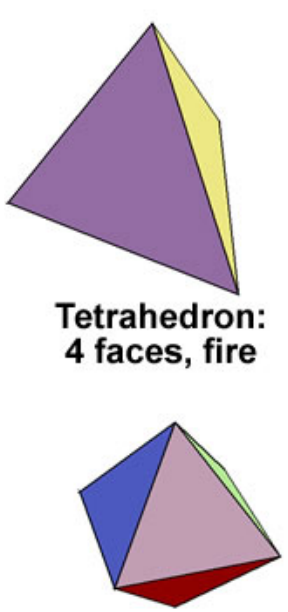

Octahedron: 8 faces, air

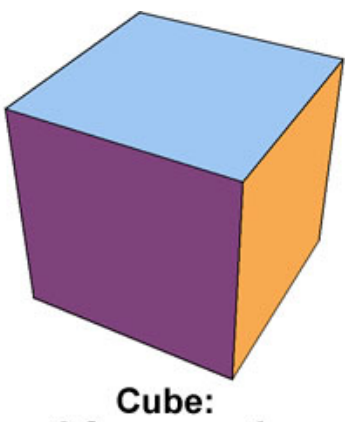

6 faces, earth

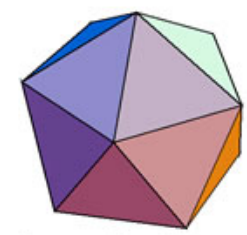

Icosahedron: 20 faces, water

The fifth regular polyhedron, the 12 faced dodecahedron, "divinity employed in the fabrication of the universe." (Lee trans.) 
Plato used this scheme to explain how the elements "are able from certain dissolutions into each other to become the sources of each other's generation." He constructed the faces of the polyhedra from two types of right triangles, isosceles and 30-60-90 degrees. However, even without taking the faces apart into these triangles we see that:

2 air $=2$ octahedra $=2 \times 8=16$ triangular faces

1 fire $=1$ tetrahedron $=1 \times 4=4$ triangular faces

Therefore: 2 air +1 fire $=20$ triangular faces $=1$ water

However, if we think about the triangles that make up the faces of the polyhedra we realize that they are only two dimensional, an idealization that can not exist in the material world. Thus these partons are also confined and unobservable.

\section{The Sankhya Hindus}

Hindu philosophy evolved an account of cosmic evolution, the Sankhya system, that had both continuum and atomistic aspects. Although some of the concepts can be traced back to the Upanishads and even the Rigveda, the oldest Sankhya text now available is the Sankhyakarika, which probably dates from the 5th century A.D.

The Sankhya idea was that there were five perceptible gross elements, the bhutas:

1. Ether (akasa) - ubiquitous, all-pervading, capable of vibration and sound.

2. Air (vayu) - light, cold, dry.

3. Fire (tejas) - light, hot, dry, luminous.

4. Water (apa) - liquid, cold, wet.

5. Earth (kshiti) - heavy, hard, dense.

The bhutas were composed of actual atoms, called paranus.

Initially, the universe was a formless undifferentiated invisible ground, the prakriti. The prakriti was composed of a completely homogenous mixture of three "reals" called the gunas:

1. Essence (sattva) - makes something perceptible by the senses; serene, calm, Vishnu.

2. Energy (rajas) - active, fiery, Brahma.

3. Mass (tamas) - inertial, dark, destructive - Shiva.

Then the God Purusa caused a disturbance in the equilibrium, and initiated creation.

The connection from continuum, the prakriti, to the atom, the paranus, is by the aggregation of the gunas into a type of parton called tanmatras. The tanmatras then combined to form the atoms. 
There were five types of tanmatras, and they correspond to the five senses:

1. Vibration - Hearing

2. Momentum - Touch

3. Energy - Sight

4. Viscous Attraction - Taste

5. Cohesive Attraction - Smell

However, the tanmatras themselves were unobservable. Thus they too are confined partons. Sastri comments, "The subtle elements [tanmatras] are not cognisable by us with our limited faculties; their distinctions, thus, not being perceived by us they are nonspecific" (p. $80-81)$.

Many Eastern religious traditions hold that enlightened beings can transcend all limitations. Here we see that belief expressed in the idea that with enlightenment the tanmatras become cognisable.

Note also that, in common with other similar classifications, there is a fifth element, the ether. This echoes Aristotle's proposal that an ether was the constituent matter of the heavens. Plato did not explicitly have such a concept, but did associate the fifth regular polyhedron with divinity.

\section{A World Not Made of Particles}

\section{The Bootstrap in Physics}

By the late 1920's it was known that in some atoms, neutrons in the nucleus will decay into a proton and an electron in a process called beta decay. For a worldview that includes elementary particles, it was natural to assume that in some sense neutrons are therefore composed of protons and electrons.

In 1932 Carl Anderson discovered the antimatter companion of the electron, the positively charged positron. Heisenberg was particularly elated: now he could similarly view the proton as being made of a neutron and a positron. In other words, the neutron and proton are made of each other. As he later wrote:

"The symmetry between proton and neutron was completely restored .... In the beginning was symmetry! This sounded like Plato’s Timaeus.” (Heisenberg, p. 132)

In this view, the so-called elementary particles are all reflections of all the other particles. This is often called the bootstrap, whose central idea is that the universe is a selfconsistent web of interrelations. As Chew remarked "To a bootstrapper the identification of a seemingly fundamental quark would constitute frustration.” (Chew, 1970). 
Instead of trying to classify the elementary particles, this approach concentrates on process, on the various interactions that can occur. It envisions the universe in terms of an infinite dynamic matrix, the Scattering Matrix, or S-Matrix. And each element of the Smatrix describes a particular nuclear reaction, not a particular nuclear particle. J.A. Wheeler began work on the S-matrix approach in the 1930's, (Wheeler 1937) and was shortly joined by Heisenberg and others

Chew (1968) commented: "Carried to its logical extreme, the bootstrap conjecture implies that the existence of consciousness, along with all other aspects of nature, is necessary for self-consistency of the whole.”

\section{Anaxagoras}

Anaxagoras is said to have been the first to introduce philosophy to Athens. Only fragments remain of his writing, but it seems reasonable to call him an early bootstrapper. For example, he wrote:

16. And since the portions of the great and the small are equal in number, thus also all things would be in everything. Nor yet is it possible for them to exist apart, but all things include a portion of everything. Since it is not possible for the least to exist, nothing could be separated, nor yet could it come into being of itself, but as they were in the beginning so they are now, all things together. And there are many things in all things, and of those that are separated there are things equal in number in the greater and the lesser. (Fairbanks, p. 245)

However, although Chew recognizes that in principle the bootstrap needs to include mind, Anaxagoras did not:

Other things include a portion of everything, but mind is infinite and selfpowerful and mixed with nothing, but it exists alone itself by itself. (Fairbanks, p. 240)

\section{Buddhist Elements}

The early Buddhists thought about the world in terms of the Hindu elements of air, earth, fire, water and ether. Sometimes the ether is omitted, and at other times it is called void. The concept of a void, emptiness, plays a crucial role in Buddhist thought. (e.g. Chang, passim) A clue to how the ether becomes void is the Heart Sutra, which says repeatedly:

Form is emptiness, emptiness is form.

The concept of void is also closely related to the development of the number zero, which took hold thousands of years ago in Northern India/Southern China. The number zero was adopted by the Arab mathematicians, who finally carried it to Europe. In Europe, 
however, resistance to its use continued through the $15^{\text {th }}$ century. (Dantzig, p. 31 - 33) One thing that "carried the day" in Europe was that it is very difficult to do arithmetic such as long division in Roman numerals, or any other representation without a placeholder like zero. For more information, see e.g. Seife.

A lovely calligraphy by Zen Master Sengai is The Universe. It should be "read" from right to left.

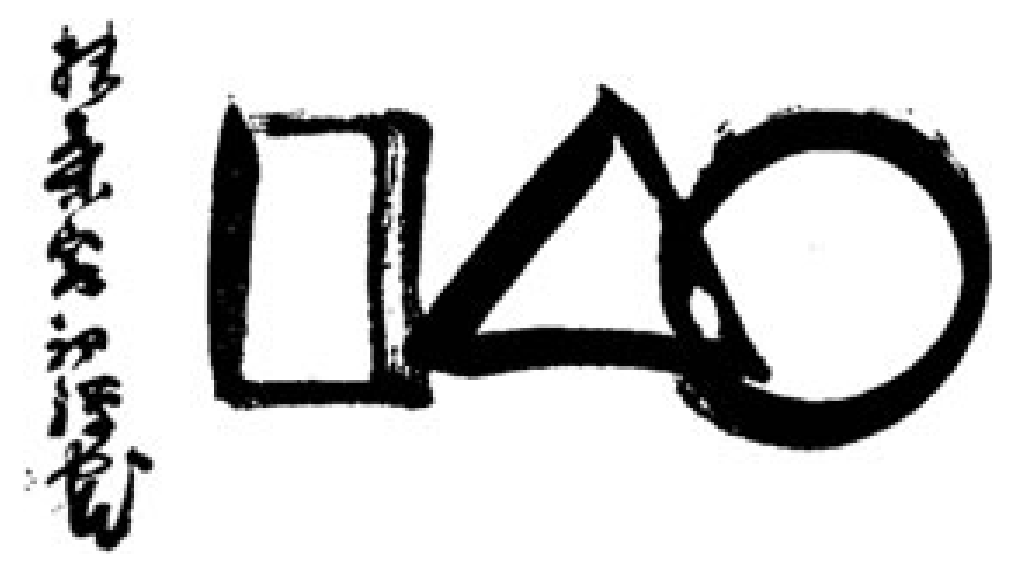

Suzuki comments:

The circle represents the infinite, and the infinite is at the basis of all beings. But the infinite in itself is formless. We humans endowed with senses and intellect demand tangible forms. Hence the triangle. The triangle is the beginning of all forms. Out of it first comes the square. The square is the triangle doubled. This doubling process goes on infinitely. (Suzuki, 1971, p. 36)

One might see an echo of the Platonic triangular partons in the calligraphy.

Consciousness is explicitly included in the scheme, since for Buddhists mind is the sixth sense, in addition to touch, sight, hearing, smell, and taste.

However, the Buddhists are not atomists. As the Lankavatara Sutra states:

Long and short and suchlike come to exist mutually conditioned; not-to-be grows effective by to-be and to-be by not-to-be.

When things are analyzed into atoms, there remains nothing to be discriminated as objects. (Suzuki, 1972, p. 175)

In fact, for the Buddhists the elements are a pure bootstrap.

It is clear that the fundamental elements are rather forces or momentary quanta of energy than substantial atoms. They accordingly fall under the category of 'cooperators' or cooperating forces. (Stcherbatsky, Vol 1, p. 101) 
A popular image in both Hindu and Buddhist thought illustrates the idea of a bootstrap very nicely: Indra's Net of Pearls.

In the heaven of Indra, there is said to be a network of pearls, so arranged that if you look at one you see all the others reflected in it. In the same way, each object in the world is not merely itself but involves every other object and in fact is everything else.

\section{Chinese Elements}

In the 4th century B.C.E. the Chinese were developing classifications that seemed to be a rudimentary form of atomism (Needham, Vol. 4, Pt. I, p. 6). However, this was dropped by a classification of the world in terms of 5 elements: wood, fire, earth, metal and water. The Chinese associated and classified everything under this scheme: the seasons, directions, musical modes, internal organs, colors, human faculties, creatures, geometric forms, and more.

Shu Ching is a collection of documents said to have been put together by Confucius. In the Great Plan chapter we find:

The nature of water is to soak and descend; of fire to blaze and ascend; of wood to be crooked and to be straight; of metal to obey and to change; while the virtue of earth is seen in seed-sowing and in gathering. (Legge, p. 325 - 326)

It seems clear that the elements do not represent five types of matter, or even qualities as with Plato and Aristotle; rather they are emblems of five types of process. Needham comments: "Chinese thought here characteristically avoided substance and clung to relation” (Vol. 2, p. 243). In other words, the Chinese elements are also a bootstrap. In fact, the character for the elements, 行 (hsing, xing), also means go or move and was originally represented as a crossroads:

In interpreting this symbol, remember to "read" from top to bottom.

In the previous sub-sub-section, I very briefly discussed the origins of the number zero and its place in Buddhist thought about the void. Zero has a similarly important place in Chinese thinking about the emptiness of Taoist mysticism (Needham, Vol. 3, p. 12). It also, of course, was part of the sophisticated algebra developed by Chinese mathematicians.

\section{Bell's Theorem}

At present the vast majority of high energy physicists are elementary particle physicists. Thus, in some sense atomism has "won" the contest. However, there are at least two threads of current thought in Physics which reflect ideas from the bootstrap. In this subsub-section I discuss one of them, and in the next the other. 
In 1964 Bell published a theorem which, when experimentally tested, shows that if there is a world separate from its observation it is necessarily non-local (Bell , Harrison). By non-local I mean that each spatially separate part of the universe is instantaneously correlated with every other part of the universe. Stapp correctly calls this "the most profound discovery of science.”

The origins of Bell's Theorem is a famous paper by Einstein, Podolsky and Rosen (EPR) published in 1935 that investigated the "spooky action-at-a-distance" that seems to be part of Quantum Mechanics. In 1951 Bohm re-formulated the EPR argument in a simpler way, and it was this form that Bell used for his theorem.

Nobody thought more deeply about the consequences of a non-local universe than Bohm (Bohm 1980 and Bohm \& Hiley 1993). Bohm called our everyday world of space, time and causality the explicate order. He proposed that underlying this everyday world is an interconnected one which he calls the implicate order. He used a number of analogies and images to discuss these two orders.

In one analogy he imagined a large cylindrical glass container of glycerine mounted on a turntable. We place a spot of black ink in the glycerine. We slowly rotate the container, and the ink gradually disperses throughout the glycerine. If we slowly rotate the cylinder in the opposite direction the spot of ink gradually re-forms. When the ink is dispersed it is in an implicate state: it exists throughout the glycerine. When the ink is a spot it is explicate: it exists in one part of the glycerine but not in the other parts. If we continue rotating the cylinder in this opposite direction the spot disperses again.

We extend the image as follows. We place the spot of ink as before. We slowly rotate the cylinder one revolution, and the ink has begun to disperse. We place a second spot of ink just beside where the first spot was, and rotate for one more revolution. A third spot is placed beside where the second was, one more revolution, and we continue this for a few spots. Then we continue slowly rotating the cylinder until all the ink is fully dispersed. When we reverse the direction of rotation we see the last spot coalesce, then the next to last one right beside the last one, and so on. We could interpret what we are seeing as a single spot of ink that is moving. So in the implicate fully dispersed state we have enfolded the motion in space and time of an object throughout the glycerine. Reversing the rotation unfolds the reality back into space and time.

Another analogy is a hologram. As shown to the right, to make a hologram we split a laser beam into two pieces with a halfsilvered mirror, a beamsplitter. One piece is reflected and goes to a photographic plate, the

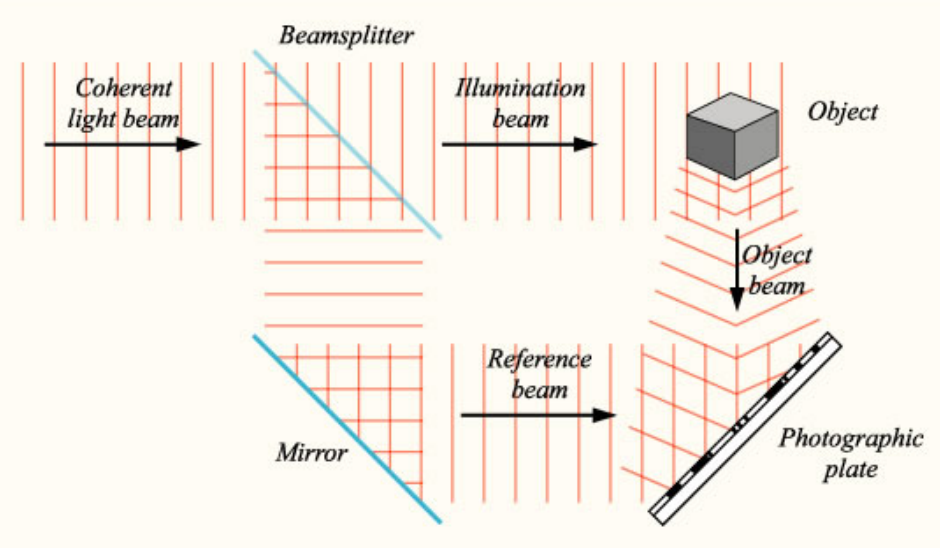


other bounces off the object and then goes to the plate. In order to reconstruct the image of the object we shine a laser beam through the developed plate: the three-dimensional image appears. Note that in some sense the hologram on the plate is an interference pattern between the beam that has experienced the thing and the beam that experienced no-thing. (Figure from Wikipedia Hologram)

One characteristic of a hologram is that down to at least a few grains of the silver in the plate, each piece of the plate contains the entire image. If we cut the plate in half we do not lose half the image; instead we lose resolution and the image becomes more fuzzy. Thus each piece of the plate contains the entire space of the object in an enfolded way; this is an analogy to the implicate order. When we reconstruct the image, we have unfolded the implicate order into an explicate one.

There are "multiplexed" holograms that contain time information too. If the object is moving, we rotate the photographic plate. When we reconstruct the image if we look from different angles we see the object's motion. Here the object's time behavior is also enfolded into the totality.

We see that in the implicate order there is no spatial or time separation. Thus it is a nonlocal order.

Here is another image used by Bohm:
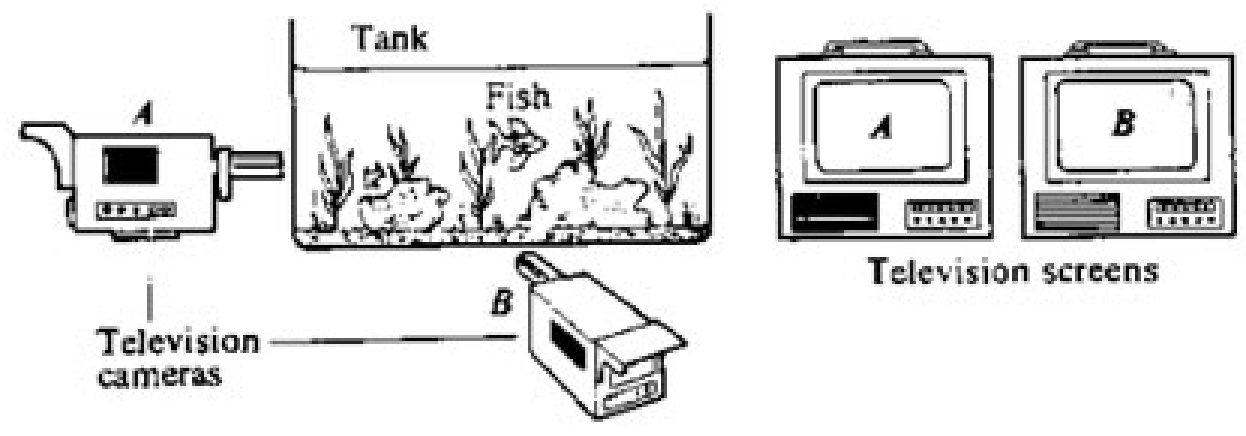

Television screens

Bohm comments:

The images on the screens are two dimensional projections (or facets) of a three dimensional reality. ... Yet, since these projections exist only as abstractions, the three-dimensional reality is neither of these. ... What is actually found [in the experimental tests of Bell's theorem] is that the behavior of the two [electrons] is correlated in a way that is rather similar to that of the two television images of the fish, as described earlier. Thus ... each electron acts as if it were a projection of a higher-dimensional reality. ... What we are proposing here is that the quantum property of a non-local, non-causal relation of distant elements may be understood through an extension of the notion described above. (p. 187 - 188)

Bohm and Hiley also considered the role of mind in the process: 
Classical physics provided a mirror that reflected only the objective structure of the human being who was the observer. There is no room in this scheme for his mental process which is thus regarded as separate or as a mere 'epiphenomenon' of the objective processes. ... [Through the] mirror [of quantum physics] the observer sees 'himself' both physically and mentally in the larger setting of the universe as a whole. ... More broadly one could say that through the human being, the universe is making a mirror to observe itself. (p. 389)

\section{String Theory}

First, one needs to realize that string theory as such does not really exist. Instead, it is a program that intends to build such a theory. Here I will only skim the surface of this exciting effort; further details can be found at The Official String Theory Web Site at http://superstringtheory.com/index.html or in Brian Greene's book The Elegant Universe.

The string theorists hope to solve a long-standing problem. We can classify the world in terms of the interactions that can occur:

1. Strong - responsible for holding the nucleus together.

2. Weak - responsible for radioactive decay.

3. Electromagnetic.

4. Gravitation.

It used to be thought that the magnetic interaction and the electric interaction were separate. They were unified by Faraday and Maxwell into the electromagnetic interaction in the 19th century. Physicists would like to continue unifying these interactions, and some progress has been made on unifying the strong, weak, and electromagnetic forces. However, our best theory of gravitation, Einstein's General Theory of Relativity, continues to stand alone, resisting all attempts at unification. String theory hopes to achieve this unification.

In string theories, as in the bootstrap, there are no particles; there are only strings and membranes. The vibrations of the strings and membranes are what we normally interpret as the elementary particles. The different modes of vibration correspond to the different particles.

The reason why the elementary objects, electrons, photons, quarks, etc., appear to us to be point-particles instead of vibrating strings is that size of the strings are much too small to be directly seen with current technology. It turns out that the dimension of the strings is much greater than the three spatial dimensions and one time dimension we are used to. However, those extra dimensions are "folded up" inside the string, so are unobservable. This is called "compactification." Currently, the dimensionality of spacetime is believed to be 10 or 11, of which only 4 are normally observable: the three spatial dimensions and the time dimension. 


\section{Monads}

If you have already read Globus' "Quantum Monadology” in this issue of NeuroQuantology, then the main conclusion of the article may already by obvious: the bootstrap and its descendants have much in common with Leibnizian thought. Here I keep the discussion fairly non-technical; more technical discussions are by Barbour and by Smolin.

A famous thought-experiment used to think about the role of consciousness in quantum measurements is Schrödinger's Cat. In its modern from it was published in 1935 in response to the EPR analysis of Quantum Mechanics that is discussed above. We think of a cat in a box with an apparatus that will kill the cat when a radioactive atom decays. At the end of one half-life the quantum mechanical description is that the living cat and the dead cat are mixed in equal proportions. When we look into the box, the "state" collapses into either a live cat or a dead cat. A review of the paradox's place in these issues is DeWitt. de Beauregard commented:

Finally, the need for consistency of the whole scheme leads me to think of the world we are living in as a Leibnitzian world, where cats are rather high in the hierarchy of monads.

Earlier I referred to Wheeler's work on the S-Matrix and the bootstrap, starting in 1937. He noticed the similarity of his work to Leibniz:

There may be no such thing as the 'glittering central mechanism of the universe' to be seen behind a glass wall at the end of the trail. Not machinery but magic may be the treasure that is waiting. Rather than Newtonian law it may resemble more the logic of relationships that Leibniz envisaged. (Wheeler 1973, p. 203)

Gale also recognized the similarity:

Leibniz constantly speaks of the monads as being in `mirroring' relations. But what are the relata of the mirroring relations? Mirrors mirroring other mirrors. Leibniz's monadic kingdom is a pure bootstrap operation.

The fact that Leibniz' monads and the Chinese elements are both bootstraps may not be coincidental or even archetypal. Leibniz' philosophy was strongly influenced by Chinese Neo-Confucian thought brought back to Europe by Jesuit missionaries. On the next page is the frontispiece of a paper by Leibniz on Chinese philosophy, one of the first such studies carried out in Europe.

Earlier I introduced a correlation of the concepts of void, emptiness and the number zero with ideas of the bootstrap in Buddhist and Chinese classifications of the world. Zero was also crucial for Leibniz, and in particular his invention of binary arithmetic, the language of the modern computer. One of the strong influences on Leibniz in developing binary arithmetic appears to have been the ancient I Ching (Needham, Vol. 2, p.341; Ryan). This 
book of wisdom, disguised as an oracle, was called by Jung "the experimental basis of Chinese philosophy.” (Jung \& Pauli, p. 49)

I close with a comment by Needham:

An unexpected vista thus opens before our eyes - the possibility that while the philosophy of fortuitous concourse of atoms, stemming from the society of European city-states, was essential for the construction of modern science in its 19th century form; the philosophy of organism essential for the construction of modern science in its present and coming form stemmed from the bureaucratic society of ancient and medieval China. (Needham, Vol. 2, p. 339)

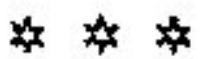

\section{LETTR XVIII. \\ DE MONS. D正 LEIBNIZ \\ SVR LA \\ PHLLOSOPHIE CHINOISE}

MONS. DE AEMOND;

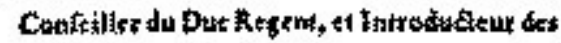

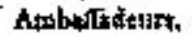

\section{SECTHON PREMIERE \\ DV SENIIMENTY DES CUINOIS 1) E DIEV.}

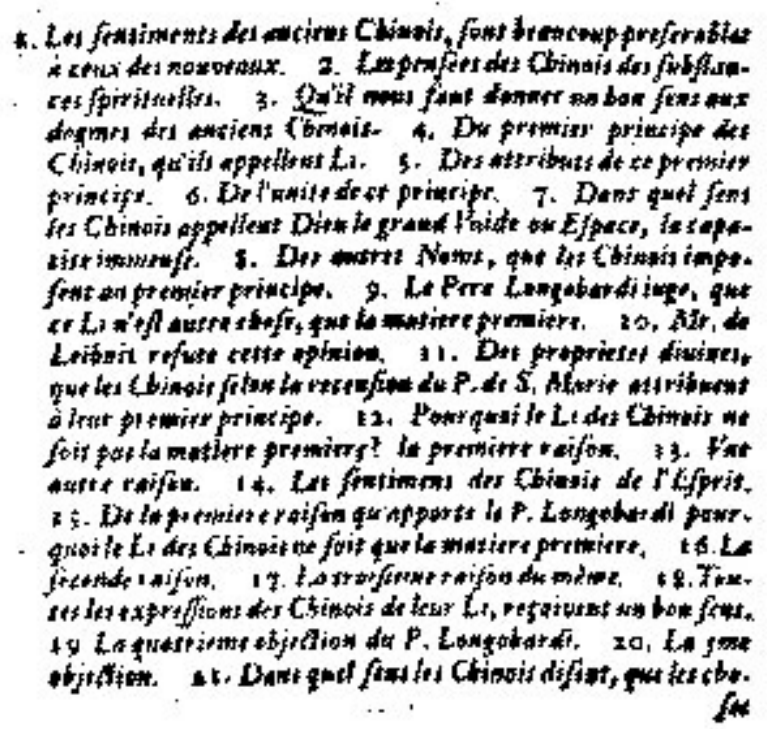




\section{References}

Barbour JB. Leibnizian time, Machian dynamics, and quantum gravity. Penrose R. and Isham CJ eds. Quantum Concepts in Space and Time: Oxford Science; 1986. p. 236 246.

Bell JS. On the Einstein Podolsky Rosen paradox. Physics 1964; 1: 195.

Bohm D. Quantum Theory. New Jersey: Prentice-Hall; 1951. p.611 - 623.

Bohm D. Wholeness and the Implicate Order. Boston: Routledge \& Kegan Paul; 1980.

Bohm D and Hiley BJ. The Undivided Universe. NY: Routledge \& Kegan Paul; 1993.

Chang GCC. The Buddhist Teaching of Totality. Univ. Park PA: Penn. State UP; 1971.

Chew GF. “Bootstrap”: A scientific idea? Science 1968; 171: 762 - 765.

Chew GF. Physics Today 1970: 23(10): 23.

de Beauregard OC. Time Symmetry and Interpretations of Quantum Mechanics.

Foundations of Physics 1976: 6(5): 539 - 556.

Dantzig T. Number: The Language of Science, $2^{\text {nd }}$ ed. London: George Allen and Unwin; 1940.

DeWitt B. Quantum mechanics and reality. Physics Today 1970: 23(9): 30 - 35.

Reprinted in DeWitt BS and Graham N, eds. The Many-World Interpretation of Quantum Mechnics. Princeton: Princeton UP; 1973. p. 155 - 166.

Einstein A, Podolsky B \& Rosen N. Can Quantum-Mechanical Description of Physical Reality Be Considered Complete? Physical Review 1935; 47: 777-780.

Fairbanks A. The First Philosophers of Greece. London: K. Paul, Trench, Trubner; 1898.

Gale G. Chew’s Monadology. Jour. History of Ideas 1974; 35: 339.

Greene B. The Elegant Universe: superstrings, hidden dimensions, and the quest for the ultimate theory. NY: Vintage; 2000.

Harrison DM. Bell's Theorem 1999, most recent revision March 2006. The html version is: http://www.upscale.utoronto.ca/PVB/Harrison/BellsTheorem/BellsTheorem.html. The pdf version is:

http://www.upscale.utoronto.ca/PVB/Harrison/BellsTheorem/BellsTheorem.pdf

Heisenberg W. Physics and Beyond. New York: Harper and Row; 1971. 
Holton G. Thematic Origins of Scientific Thought: Kepler to Einstein. Cambridge MA: Harvard; 1973.

Humphries R. The Way Things Are. Bloomington IN: Indiana UP; 1969.

Gaisser JH \& Gaisser TK. Partons in antiquity. American Journal of Physics 1977; 45: $439-442$.

Jung CG \& Pauli W. The Interpretation of Nature and the Psyche. NY: Pantheon; 1955.

Legge J. The Chinese Classics. Hong Kong: Hong Kong UP; 1960.

Needham J. Science and Civilisation in China. Cambridge: Cambridge UP; 1954 - 2004.

Plato. Timaeus, Lee HDP trans. Baltimore: Penguin; 1965.

Ryan J. Leibniz’ Binary System and Shao Yong's “Yijing”. Philosophy East and West 1996; 46: 59 - 90. Available on JSTOR at http://www.jstor.org/journals/00318221.html retrieved 2006-07-30.

Sastri SSS. Sankhyakarika of Isvara Krsna. Madras: Univ. of Madras Press; 1973.

Seife C. Zero: The Biography of a Dangerous Idea. NY. Viking; 2000.

Smolin L. The case for background independence; 2006.

http://arxiv.org/abs/hep-th/0507235. Retrieved 2006-08-5.

Stapp HP. Theory of Reality. Foundations of Physics 1977; 7: 313.

Stcherbatsky FT. Buddhist Logic. NY: Dover; 1962. Originally published in Leningrad circa 1930.

Suzuki DT. Studies in the Lankavatara Sutra. Boston: Routledge and Kegan Paul; 1972.

Suzuki DT. Sengai the Zen Master. London: Faber and Faber; 1971.

von Franz ML. Number and Time. London: Rider; 1974.

Wheeler JA. On the Mathematical Description of Light Nuclei by the Method of Resonating Group Structure. Physical Review 1937; 45: 1107.

Wheeler JA. From Relativity to Mutability. In Mehra J (ed). The Physicist's Conception of the Universe. Boston: Reidel; 1973. p. $202-250$. 
Wikipedia Hologram page. http://en.wikipedia.org/wiki/Holography Retrieved 2006-07$\underline{29}$.

Note: this is an invited article that appeared in the e-journal Neuroquantology 2006; 4(3): $252-262$. 